Original Article

\title{
Detection of specific bacterial agents by quantitative PCR assays in the bronchoalveolar lavage fluid of dogs with eosinophilic bronchopneumopathy vs. dogs with chronic bronchitis and healthy dogs
}

\author{
A.M. Canonne ${ }^{\mathrm{a}, 1, *}$, I. Peters $^{\mathrm{b}}$, E. Roels ${ }^{\mathrm{c}}$, L. Desquilbet $^{\mathrm{d}}$, C. Clercx $^{\mathrm{c}}$ \\ a Internal Medicine Unit, National Veterinary School of Alfort, 94700 Maisons-Alfort, France \\ ${ }^{\mathrm{b}}$ Torrance Diamond Diagnostic Services, The Innovation Centre University of Exeter, EX4 4RN Exeter, UK \\ ${ }^{\mathrm{c}}$ Department of Clinical Sciences, Faculty of Veterinary Medicine, University of Liège, 4000 Liège, Belgium \\ d Unit of Biostatistics, National Veterinary School of Alfort, 94700 Maisons-Alfort, France
}

A R T I C L E I N F O

Article history:

Accepted 16 December 2017

\section{Keywords:}

Bordetella bronchiseptica

Bronchoalveolar lavage

Dogs

Eosinophilic bronchopneumopathy

Mycoplasma spp.

\begin{abstract}
A B S T R A C T
In humans, Mycoplasma pneumoniae and Bordetella pertussis infections are suggested to trigger or exacerbate asthma. Whether Mycoplasma or Bordetella are associated with chronic inflammatory bronchial diseases in dogs has not been investigated. The aim of this study was to assess detection rates of Mycoplasma canis (M. canis), M. cynos and Bordetella bronchiseptica (Bb), in dogs with eosinophilic bronchopneumopathy (EBP) and chronic bronchitis (CB), compared with healthy dogs. Specific quantitative PCR (qPCR) analysis for $M$. canis, $M$. cynos and $B b$ were retrospectively performed on bronchoalveolar lavage fluid (BALF) collected from 24 dogs with EBP, 21 dogs with CB and 15 healthy dogs. Possible associations between qPCR results and age, BALF cytology or clinical severity scores (CSS) in dogs with EBP were investigated.

There was no difference in M. canis, M. cynos and Bb detection rates in dogs with $\operatorname{EBP}(n=6, n=2$ and $n=6$, respectively) and dogs with $\mathrm{CB}(n=2, n=2$ and $n=2$, respectively) compared with control dogs ( $n=4, n=2$ and $n=2$, respectively). In dogs with EBP, the proportion that were qPCR-positive for $B b$ was higher in dogs with higher CSS $(P=0.014)$ and BALF from $B b$-positive dogs had higher percentage of neutrophils $(P<0.001)$. Among dogs that were qPCR-positive for $B b$, moderate to high loads were only detected in dogs with EBP. M. canis and $M$. cynos detection was not associated with EBP or CB; higher $B b$ loads were only present in dogs with EBP and high CSS. A possible cause and effect relationship between $B b$ infection or load and EBP remains unclear and requires further investigation.
\end{abstract}

(c) 2017 Elsevier Ltd. All rights reserved.

\section{Introduction}

Idiopathic eosinophilic bronchopneumopathy (EBP) is a chronic disease characterized by eosinophilic infiltration of the lung and bronchial mucosa in young adult dogs (Corcoran et al., 1991; Clercx et al., 2000; Rajamäki et al., 2002). Dogs with non-specific chronic bronchitis (CB) also present with chronic cough, but are usually older. The etiology of both inflammatory conditions remains unclear. In EBP, an underlying immunologic hypersensitivity is

\footnotetext{
* Corresponding author.

E-mail address: morgane.canonne-guibert@vet-alfort.fr (A.M. Canonne).

1 Present address: Internal Medicine Unit, National Veterinary School of Alfort, 7 Avenue Charles de Gaulles, 94700 Maisons-Alfort, France.
}

suspected, but the inciting antigens remain mostly unidentified (Clercx et al., 2002; Peeters et al., 2005; Clercx and Peeters, 2007). For both of these bronchial diseases, current treatments consist of long-term oral and/or inhaled glucocorticoids (Clercx et al., 2000; Bexfiels et al., 2006; Rozanski, 2014; Canonne et al., 2016b).

In humans, infections with Mycoplasma pneumoniae ( $M$. pneumoniae) have been associated with asthma for decades (Hansbro et al., 2004; Atkinson, 2013; Ye et al., 2014). M. pneumoniae infection is associated with acute exacerbation of adult asthmatics and future development of asthma in children (Hansbro et al., 2004) and specific treatment improves pulmonary function in asthmatics (Kraft et al., 2002). However, Bordetella pertussis (B. pertussis) has been also discussed as potential trigger in human inflammatory bronchial disease and asthma (Harju et al., 2006; Wakashin et al., 2008; Nicolai et al., 2013; Yin et al., 2017). 
Furthermore, in a mouse model of allergic asthma, prior B. pertussis infection was documented to exacerbate airway signs (Ennis et al., 2004; Kavanagh et al., 2010).

Although Mycoplasma cynos (M. cynos) has been identified as an emerging, possibly contagious, and lethal pathogen in dogs with canine infectious respiratory disease (CIRD, kennel cough or canine infectious tracheobronchitis; Rycroft et al., 2007; Zeugswetter et al., 2007; Mannering et al., 2009; Priestnall et al., 2014), but the exact role of $M$. canis and $M$. cynos as primary respiratory pathogens still remains unclear (Chandler and Lappin, 2002; Chalker et al., 2004; Chan et al., 2013). Moreover, a recent study demonstrated the impact of oral bacterial contamination on Mycoplasma-specific PCR results of bronchoalveolar lavage fluid (BALF; Chan et al., 2013), making the investigation of a potential primary role of Mycoplasma spp. in canine lower respiratory conditions even more complex.

Bordetella bronchiseptica $(B b)$ is recognized as one of the primary causative pathogen agents of CIRD. $B b$ can exist in respiratory tract of dogs as either commensal or pathogen (Bemis et al., 1977; Bemis, 1992; Schulz et al., 2014). Currently, quantitative PCR ( $\mathrm{qPCR}$ ) assays provide highly sensitive and specific detection of of Bb, M. canis and M. cynos (Chan et al., 2013; Schulz et al., 2014; Lavan and Knesl, 2015; Canonne et al., 2016a). However, the potential role of these infectious agents in chronic inflammatory bronchial diseases in dogs have not yet been investigated and whether these bacterial agents could act as triggers or exacerbating agents in dogs with EBP is unknown.

The aim of the present study was to evaluate the presence and bacterial load of $M$. canis, $M$. cynos and $B b$ in steroid-naïve dogs newly-diagnosed with EBP and compare the results to those for dogs with non-specific CB and healthy dogs, using specific qPCR analysis performed on BALF samples. Additionally, potential associations between positive qPCR results for any of these bacterial species and the clinical severity of EBP were assessed.

\section{Materials and methods}

Dogs

Client-owned dogs presented to the National Veterinary School of Alfort Companion Animal Hospital between March 2009 and February 2016 and diagnosed with EBP or CB were prospectively recruited.

Definitive diagnosis of idiopathic EBP and CB was made in accordance with criteria previously described (Clercx et al., 2000; Rozanski, 2014). Briefly, this was based on compatible respiratory clinical complaints, radiological changes, bronchoscopic findings, analysis of the BALF including culture and standard cytological examination and, in some cases, histopathological examination of endoscopic bronchial mucosal biopsies. In dogs with $\mathrm{CB}$, bacterial involvement in neutrophilic airway inflammation was excluded based on cytology (lack of degenerative neutrophils, absence of intra-cellular bacteria), culture and positive response to steroids. In dogs with EBP, exclusion of other causes of eosinophilic airway inflammation, including cardiopulmonary parasites, was required to definitively diagnose idiopathic EBP by using fecal and BALF analysis (Baermann method, cytology and qPCR on BALF). All dogs were newly-diagnosed and steroidnaïve.

In dogs with a final diagnosis of idiopathic EBP, a clinical severity score (CSS; 1 $5 / 5$ ) was assessed at the time of diagnosis based on severity and frequency of cough, evidence of exercise intolerance or lethargy, as previously defined (Canonne et al., 2016b). Cough was scored from 1-3/3 depending on frequency and severity; presence of retching or exercise intolerance was recorded with 1 additional point Points obtained from cough scoring, retching and exercise intolerance were added to a maximum total of 5 points.

Bronchoscopy, BALF procedure and processing were performed as described earlier (Clercx et al., 2000). Briefly, dogs were anesthetized using various anesthetic protocols after a $5 \mathrm{~min}$ pre-oxygenation period. Five to $20 \mathrm{~mL}$ aliquots (depending on bodyweight) of sterile saline $(\mathrm{NaCl} 0.9 \%)$ were instilled twice into a same bronchus and a third time into a different lung lobe via a flexible pediatric endoscope (Fujinon, Pediatric video-bronchoscope EB-530S), followed by immediate aspiration by gentle automated suction. The recovered BALF was immediately processed. Aliquots of naïve BALF were used for semi-quantitative bacterial culture (Collard Laboratories, according to methods previously described; Peeters et al. 2000), total cell count determination using a hemocytometer, and cytospin preparation (centrifugation at $1400 \mathrm{rpm}, 197 \mathrm{~g}$, for $4 \mathrm{~min}$ at $20^{\circ} \mathrm{C}$, Thermo Shandon Cytospin 4). Differential cell count calculations were established by counting a total of 300 cells viewed on high power fields on the cytospin preparation; 400-600/ $\mathrm{L}$ was considered a normal cell count. BALF cytology was considered normal if there were $<12 \%$ neutrophils and $<7 \%$ eosinophils. The remaining recovered BALF was centrifuged at $1300 \mathrm{~g}$ for $15 \mathrm{~min}$ at $4{ }^{\circ} \mathrm{C}$. The resulting supernatant and cell pellet were stored separately at $-80^{\circ} \mathrm{C}$. Additionally, angiostrongylosis was excluded in all dogs with EBP based on a negative quantitative PCR (qPCR) test results in BALF (Canonne et al., 2016b). Additionally, all dogs were treated at the time of diagnosis with anthelmintics (fenbendazole $50 \mathrm{mg} / \mathrm{kg}$ q24h for 5 days, Panacur Intervet International via MSD Animal Health, or milbemycin oxime $1 \mathrm{mg} / \mathrm{kg}$ once, Milbemax Novartis), while results were pending.

\section{Control group}

Stored BALF samples collected from 15 healthy dogs were available from previous studies for which ethical approval had been previously obtained from the University Local Ethical Committee (University Local Ethical Committee Approval number 1435; Approval date 30th April 2013). The stored samples were collected from healthy dogs belonging to veterinary staff or students $(n=13)$ or from shelters $(n=2)$, and none had either history or clinical signs of respiratory disease. Bronchoscopy, BALF and laboratory processing and storage had been performed as for dogs with EBP and CB and both total cell counts and cytological examination of cytocentrifuged preparations of BALF were within normal limits in each dog.

\section{Quantitative PCR analysis}

Quantitative PCR analysis was performed on stored BALF for M. canis, M. cynos and $B b$ in dogs with EBP, $C B$ and healthy dogs. The frozen pelleted cells were thawed and, after re-suspension in a small volume of sterile saline $(0.9 \% \mathrm{NaCl})$, samples were sent to the laboratory for qPCR analysis. The qPCR results were expressed as $\mathrm{Ct}$ values. Ct values were arbitrarily further categorized into five groups: very high load $(\mathrm{Ct}<20)$, high load (20.1-24), moderate load (24.1-28), low load (Ct 28.1-32) and very low load $(>32.1)$.

Statistical analysis

Statistical analyses were performed with a commercially available software (XLstat software). Data were expressed for continuous and categorical variables as median with range or as proportions, respectively.

Chi-square or Fisher's exact (when necessary) tests were used to compare the proportions of qPCR detection of $M$. canis, $M$. cynos and $B b$ between dogs with EBP and healthy dogs and between dogs with EBP and dogs with CB. Dogs with EBP were grouped according to CSS into two groups: dogs with CSS $\leq 3$ (mild clinical severity) and dogs with CSS $>3$ (moderate-to-severe clinical signs). The proportion of dogs with EBP and positive qPCR results for $M$. canis, $M$. cynos and $B b$ were compared between dogs with CSS $\leq 3$ and dogs with CSS $>3$ by using exact Fisher's exact test (for $n \leq 5$ ). For each bacterial agent, the median age of dogs with EBP with positive qPCR was compared to the median age of dogs with EBP with negative qPCR using Wilcoxon-Mann Whitney tests. For each bacterial agent, median total cell count and percentage of neutrophils in the BALF of dogs with EBP that were qPCRpositive were compared to BALF parameters from dogs with EBP that were qPCRnegative by using Wilcoxon-Mann Whitney's test.

Values of $P \leq 0.05$ were considered statistically significant.

\section{Results}

A convenience sample of 24 dogs with EBP (median age, $4.2 \mathrm{y}$ [0.9-13.2]) and 21 dogs with non-specific CB (median age, 8.0 [0.914.0]) were selected.

Table 1 reports the proportions of dogs with positive qPCR result for $M$. canis, $M$. cynos and $B b$ in each of the three groups. For each bacterial agent, no significant difference in qPCR detection rates were found between dogs diagnosed with EBP and healthy dogs and between dogs with EBP and dogs with CB (Table 1). Among dogs with positive qPCR for $M$. cynos on BALF, Ct values corresponding to very high load were only found in dogs with inflammatory bronchial disease. Among dogs with positive qPCR for $B b$ on BALF, moderate or high load were only found in dogs with EBP.

In dogs with EBP, CSS varied between two and five at the time of diagnosis; total BALF cell counts were $700-6000 / \mathrm{mL}$; \% eosinophils was 20-85; and \% neutrophils was 1-30 (Table 2). The proportion of dogs with $B b$ in the BALF was significantly higher in dogs with CSS $>3(5 / 9,56 \%)$ than in dogs with $\operatorname{CSS} \leq 3(1 / 15,7 \% ; P=0.015)$. 
Table 1

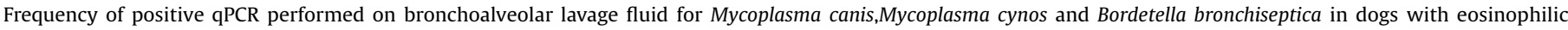
bronchopneumopathy (EBP), chronic bronchitis (CB) and healthy dogs.

\begin{tabular}{|c|c|c|c|c|}
\hline & Dogs with EBP & Dogs with $C B$ & Healthy dogs & $P$ \\
\hline Mycoplasma canis & $6 / 24(25 \%)^{a, b}$ & $2 / 21(10 \%)^{\mathrm{b}}$ & $4 / 15(13 \%)^{a}$ & $\begin{array}{l}1.00^{\mathrm{a}} \\
0.25^{\mathrm{b}}\end{array}$ \\
\hline Very high load $(\mathrm{Ct}<20)$ & - & - & & \\
\hline High load $(20.1-24)$ & - & - & & \\
\hline Moderate load (24.1-28) & 1 & - & & \\
\hline Low load (Ct 28.1-32) & 3 & - & 1 & \\
\hline Very low load $(>32.1)$ & 2 & 2 & 3 & \\
\hline Mycoplasma cynos & $2 / 24(8 \%)^{a, b}$ & $2 / 21(10 \%)^{b}$ & $2 / 15(13 \%)^{a}$ & $\begin{array}{l}0.63^{\mathrm{a}} \\
1.00^{\mathrm{b}}\end{array}$ \\
\hline Very high load $(\mathrm{Ct}<20)$ & 2 & 1 & - & \\
\hline High load (20.1-24) & - & - & - & \\
\hline Moderate load (24.1-28) & - & - & 1 & \\
\hline Low load (Ct 28.1-32) & - & - & - & \\
\hline Very low load $(>32.1)$ & - & 1 & 1 & \\
\hline Bordetella bronchiseptica & $6 / 24(25 \%)^{a, b}$ & $2 / 21(10 \%)^{\mathrm{b}}$ & $2 / 15(13 \%)^{a}$ & $\begin{array}{l}0.45^{\mathrm{a}} \\
0.25^{\mathrm{b}}\end{array}$ \\
\hline Very high load $(\mathrm{Ct}<20)$ & - & - & - & \\
\hline High load (20.1-24) & 1 & - & - & \\
\hline Moderate load (24.1-28) & 2 & - & - & \\
\hline Low load (Ct 28.1-32) & - & - & - & \\
\hline Very low load $(>32.1)$ & 3 & 2 & 2 & \\
\hline
\end{tabular}

${ }^{\mathrm{a}, \mathrm{b}} \mathrm{Groups}$ with same letter were compared.

For the mycoplasmal species, there was no difference in CSS between dogs with qPCR-positive qPCR-negative results (data not shown). In dogs with EBP, the median age of those that were qPCRnegative for $B b$ was not different from that for qPCR-positive dogs (3.7 years [0.9-13.0] and 5.8 years [0.9-13.2], respectively; $P=0.44$ ) and median age for qPCR-negative and qPCR-positive for $M$. canis and $M$. cynos was not different ( $M$. canis, 4.3 years $[0.9-13.0]$ and 2.9 years [1.6-13.2], respectively; $P=0.73 ; M$. cynos, 4.1 years [0.9-13.0] and 7.6 years [0.9-13.2], respectively; $P=0.71$ ).

In dogs with EBP, for each bacterial agent taken separately, median BALF total cell counts from qPCR-negative dogs was not different from that from qPCR-positive dogs (data not shown). Median \% neutrophils in BALF from dogs qPCR-positive for $B b$ and $M$. cynos were significantly higher than median values from dogs that were qPCR-negative $(B b, 15 \%[3-20]$ and $3.5 \%$ [1-30], respectively; $P<0.001 ; M$. cynos, $12.5 \%(10-15)$ and $3.5 \%$ [1-30], respectively; $P=0.02$ ). Median \% neutrophils in BALF from dogs with positive and negative qPCR results for $M$. canis were not different (data not shown).

\section{Discussion}

The objectives of the present study were to detect the presence and load of $M$. canis, $M$. cynos and $B b$ in dogs with EBP compared with dogs diagnosed with non-specific $\mathrm{CB}$ and healthy controls, using specific qPCR on BALF samples. The results of the present study suggest that any association between either mycoplasmal species and canine EBP is unlikely. However, in dogs with EBP, the probability of a positive qPCR result for $B b$ increased statistically with clinical severity. Moreover, among dogs with positive qPCR results for $M$. cynos or $B b$, Ct values indicating moderate, high or very high load were found only in dogs with inflammatory bronchial disease. The small group size of dogs that were qPCRpositive precluded statistical comparisons of bacterial load between groups.

Human asthma and canine EBP have different characteristics; for example, bronchial hyper-responsiveness is not a hallmark of canine EBP. However, human asthma and canine EBP are both TH2driven inflammatory conditions characterized by eosinophilic infiltration. The role of bacterial genera known to be implicated in the induction or exacerbation of human asthma have not been investigated in dogs with EBP. In humans, infection with
M. pneumoniae can favour the development of asthma in predisposed patients and specific treatment with clarithromycin improves pulmonary function (Martin et al., 2001; Kraft et al., 2002; Hansbro et al., 2004; El Sayed Zaki et al., 2009; Atkinson, 2013; Ye et al., 2014). The proportion of asthmatics who have both IgM and IgG for M. pneumoniae increases with clinical severity (Iramain et al., 2016; Yin et al., 2017). In a mouse model, single exposure to a specific toxin of $M$. pneumoniae was shown to be sufficient to cause asthma-like disease with histological evidence of pulmonary eosinophilic inflammation (Medina et al., 2012). Our study failed to support the hypothesis of a similar association between mycoplasmal infection or carriage and EBP in dogs. This could possibly be explained by the relatively small sample size limiting the power of statistical tests for comparison. In human asthma, the role of Mycoplasma spp. has sometimes been investigated using PCR, but is mainly assessed using serum Mycoplasma-specific antibodies (El Sayed Zaki et al., 2009; Iramain et al., 2016); such antibodies were not evaluated in our study. Additionally, the potential therapeutic benefit of a specific antibiotic treatment, such as doxycycline, macrolide or fluoroquinolone, has not been evaluated in dogs with EBP or $\mathrm{CB}$ and positive qPCR results for $M$. cynos. Lastly, involvement of other mycoplasmal species in canine chronic lower airway inflammation could not be excluded; several other Mycoplasma species have been investigated in dogs (Chalker et al., 2004), but until recently, accurate diagnostic methods such as qPCR were not available for less common canine mycoplasmal species.

Although $B b$ was not more frequently detected in dogs with EBP than in dogs with $C B$ or healthy dogs, the frequency of $B b$-positive dogs with EBP was positively associated with clinical severity. Among dogs with positive qPCR for $B b$ on BALF, moderate or high loads were found in dogs with EBP only. Whether $B b$ is able to trigger eosinophilic lower airway inflammation and whether the inflammatory context in airways favours $B b$ growth in dogs is unknown. In humans, $B b$ infections are unusual and almost exclusively described in immunocompromized patients with contact with animals. $B$. pertussis is more common and has been discussed as a triggering and exacerbating factor in chronic inflammatory bronchial diseases; $B$. pertussis toxin favours eosinophilic airway inflammation (Ennis et al., 2004; Harju et al., 2006; Wakashin et al., 2008; Kavanagh et al., 2010; Nicolai et al., 2013; Yin et al., 2017). Despite a large overlap between values, 
Table 2

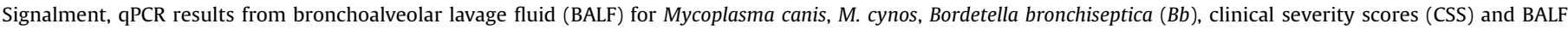
cytology in dogs with eosinophilic bronchopneumopathy (EBP).

\begin{tabular}{|c|c|c|c|c|c|c|c|c|c|}
\hline Case & Breed & Sex & $\begin{array}{c}\text { Age } \\
\text { (years) }\end{array}$ & M. canis load & M. cynos load & Bb load & CSS & $\begin{array}{c}\text { BALF cytology } \\
\text { (total cell count } / \mu \mathrm{L}, \\
\% \text { eosinophils-\% neutrophils) }\end{array}$ & BALF bacterial culture \\
\hline 1 & Mixed-breed & M & 13 & - & - & - & 2 & $\begin{array}{c}1400 / \mu L \\
20 \%-5 \%\end{array}$ & Negative \\
\hline 2 & Mixed-breed & $\mathrm{F}$ & 6 & - & - & - & 2 & $\begin{array}{c}4000 / \mu \mathrm{L} \\
85 \%-3 \%\end{array}$ & Negative \\
\hline 3 & Shar pei & $\mathrm{F}$ & 3 & - & - & - & 2 & $\begin{array}{c}2500 / \mu \mathrm{L}, \\
65 \%-5 \%\end{array}$ & Negative \\
\hline 4 & Shi tzu & M & 4 & - & - & - & 3 & $\begin{array}{c}3000 / \mu \mathrm{L} \\
60 \%-1 \%\end{array}$ & Negative \\
\hline 5 & Shi tzu & M & 1.2 & - & - & - & 3 & $\begin{array}{l}1600 / \mu \mathrm{L} \\
55 \%-30 \%\end{array}$ & Negative \\
\hline 6 & Mixed-breed & M & 12.4 & - & VH & - & 3 & $\begin{array}{l}1900 / \mu L \\
30 \%-10 \%\end{array}$ & Negative \\
\hline 7 & Beauceron & $\mathrm{F}$ & 1.6 & $\mathrm{~L}$ & - & - & 3 & $\begin{array}{c}1800 / \mu \mathrm{L} \\
55 \%-3 \%\end{array}$ & Negative \\
\hline 8 & Shar pei & $\mathrm{F}$ & 13.2 & M & - & M & 3 & $\begin{array}{c}2000 / \mu \mathrm{L} \\
55 \%-3 \%\end{array}$ & Negative \\
\hline 9 & Mixed-breed & $\mathrm{F}$ & 3.4 & - & - & - & 3 & $\begin{array}{c}1400 / \mu \mathrm{L}, \\
60 \%-1 \%\end{array}$ & Negative \\
\hline 10 & Tervuren & M & 2.0 & VL & - & - & 3 & $\begin{array}{c}700 / \mu \mathrm{L} \\
55 \%-16 \%\end{array}$ & Negative \\
\hline 11 & Whippet & $\mathrm{F}$ & 3.0 & $\mathrm{~L}$ & - & - & 3 & $\begin{array}{l}1100 / \mu \mathrm{L} \\
20 \%-3 \%\end{array}$ & Negative \\
\hline 12 & Golden retriever & $\mathrm{F}$ & 3.3 & - & - & - & 3 & $\begin{array}{c}1300 / \mu \mathrm{L} \\
80 \%-4 \%\end{array}$ & Negative \\
\hline 13 & Brittany spaniel & $\mathrm{F}$ & 7.1 & VL & - & - & 3 & $\begin{array}{l}800 / \mu \mathrm{L} \\
25 \%-3 \%\end{array}$ & Negative \\
\hline 14 & Siberian husky & M & 0.9 & - & - & - & 3 & $\begin{array}{c}1500 / \mu L \\
80 \%-3 \%\end{array}$ & Negative \\
\hline 15 & Mixed-breed & $\mathrm{F}$ & 5.2 & - & - & - & 3 & $\begin{array}{c}4000 / \mu \mathrm{L} \\
85 \%-7 \%\end{array}$ & Negative \\
\hline 16 & Doberman & M & 0.9 & - & - & VL & 4 & $\begin{array}{l}2000 / \mu L \\
40 \%-10 \%\end{array}$ & Negative \\
\hline 17 & Siberian husky & $\mathrm{F}$ & 1.0 & - & - & - & 4 & $\begin{array}{l}1600 / \mu \mathrm{L}, \\
75 \%-15 \%\end{array}$ & Negative \\
\hline 18 & Basset artesien & M & 4.3 & - & - & - & 4 & $\begin{array}{c}2500 / \mu \mathrm{L}, \\
35 \%-3 \%\end{array}$ & Negative \\
\hline 19 & Whippet & $\mathrm{F}$ & 8 & - & - & - & 4 & $\begin{array}{l}1700 / \mu \mathrm{L} \\
40 \%-15 \%\end{array}$ & Negative \\
\hline 20 & Brittany spaniel & $\mathrm{F}$ & 5.7 & - & - & M & 5 & $\begin{array}{l}2500 / \mu \mathrm{L}, \\
70 \%-20 \%\end{array}$ & Negative \\
\hline 21 & Border terrier & M & 2.9 & $\mathrm{~L}$ & $\mathrm{VH}$ & $\mathrm{H}$ & 5 & $\begin{array}{l}6000 / \mu L \\
20 \%-15 \%\end{array}$ & Negative \\
\hline 22 & Fox terrier & $\mathrm{F}$ & 10.0 & - & - & VL & 5 & $\begin{array}{l}1800 / \mu \mathrm{L} \\
58 \%-20 \%\end{array}$ & Negative \\
\hline 23 & Jack Russell terrier & M & 4.4 & - & - & - & 5 & $\begin{array}{c}3100 / \mu \mathrm{L}, \\
62 \%-1 \%\end{array}$ & Negative \\
\hline 24 & Tervueren & M & 6 & - & - & VL & 5 & $\begin{array}{l}4500 / \mu \mathrm{L} \\
60 \%-15 \%\end{array}$ & Negative \\
\hline
\end{tabular}

M, male; F, female; VH, very high load; H, high load; M, moderate load; L, low load; VL, very low load (see details in text and Table 1).

the median \% neutrophils in the BALF was higher in dogs with EBP with positive qPCR for $B b$ than in dogs with EBP with negative qPCR results, while median total cell counts were not different. Consequently, larger studies investigating the role of $B b$ in dogs with EBP and potential clinical benefits of treatment for $\mathrm{Bb}$ positive dogs are needed.

The potential influence of any previous $B b$ vaccination on positive samples could not be completely ruled out in our study. However, among dogs with known vaccination status, the number of vaccinated dogs was not different among the three groups (data not shown). In puppies vaccinated with a single dose of modified live intranasal vaccine, $B b$ was detected by qualitative PCR during 1 month after vaccination (Ruch-Gallie et al., 2016). To our knowledge, the impact of previous vaccination on qPCR performed on BALF has not been investigated. Nevertheless, none of the dogs in our study were vaccinated within 2 months prior to diagnosis.
Because of this, we assumed that the impact of previous vaccination on qPCR for $B b$ in BALF was minimal.

As all dogs with EBP and CB were referred cases, the impact of previous antimicrobial treatment, such as doxycycline or fluoroquinolones, on our qPCR results cannot be completely ruled out; this might have caused underestimates of the presence of each agent in diseased dogs compared to healthy dogs. This potential risk was considered quite low since there was usually a wash-out period of at least 2 days before BAL procedures.

The last minor limitation of this study was the lack of validation of CSS that was used to score clinical signs in dogs with EBP. To limit variability, CSS was attributed to each dog with EBP by only one author (AMC), who remained masked to qPCR results. Intra- and inter-observer variabilities in CSS evaluation should be assessed before this scoring system can be used on a larger scale in dogs with EBP. 


\section{Conclusions}

The present investigation by qPCR on BALF does not support any role for $M$. canis and $M$. cynos in the pathogenesis of EBP in dogs. Nevertheless, in dogs with EBP, $B b$ was detected more frequently in those with more severe clinical signs. Moreover, among dogs that were qPCR-positive for $B b$, moderate or high loads were observed in only dogs with EBP. Despite both observations, a cause and effect relationship between the presence and load of $B b$ and severe canine EBP remains unclear. However, since dogs with EBP could potentially act as $B b$ carriers and a source of infection for susceptible dogs, screening for this pathogen should be performed in dogs newly-diagnosed with EBP, particularly in cases where the clinical presentation is severe, there is a high percentage of neutrophils in the BALF, or if there has been an acute exacerbation of clinical signs in previously diagnosed cases. These $B b$-positive dogs should be appropriately accordingly and respiratory signs should be monitored closely.

\section{Conflict of interest statement}

None of the authors of this paper has a financial or personal relationship with other people or organisations that could inappropriately influence or bias the content of the paper.

\section{Acknowledgements}

This research did not receive any specific grant from funding agencies in the public, commercial or not-for-profit sectors. We gratefully thank Dr Maud Girod, Dr Emilie VanGrinsven, Dr Elodie Darnis, Dr Elodie Roels, Dr Valérie VanDerVorst, Dr Charlotte Auquier, Dr Emilie Krafft, Dr Frédéric Billen and veterinary technicians for their help in the inclusion of cases and the collection of samples.

\section{References}

Atkinson, T.P., 2013. Is asthma an infectious disease? New evidence. Current Allergy and Asthma Reports 13 (December), 702-709.

Bemis, D.A., Greisen, H.A., Appel, M.J., 1977. Pathogenesis of canine bordetellosis. Journal of Infectious Diseases 135, 753-762.

Bemis, D.A., 1992. Bordetella and Mycoplasma respiratory infections in dogs and cats. Veterinary Clinics of North America: Small Animal Practice 22, 1173-1186.

Bexfiels, N.H., Foale, R.D., Davison, L.J., Watson, P.J., Skelly, B.J., Herrtage, M.E., 2006. Management of 13 cases of canine respiratory disease using inhaled corticosteroids. Journal of Small Animal Practice 47, 377-382.

Canonne, A.M., Billen, F., Tual, C., Ramery, E., Roels, E., Peters, I., Clercx, C., 2016a. Quantitative PCR and cytology of bronchoalveolar lavage fluid in dogs with Bordetella bronchiseptica infection. Journal of Veterinary Internal Medicine 30, 1204-1209.

Canonne, A.M., Billen, F., Peeters, D., Bolen, G., Roels, E., Clercx, C., 2016b. Long term follow up in dogs with idiopathic eosinophilic bronchopneumopathy treated with inhaled steroid therapy. Journal of Small Animal Practice 57, 537-542.

Chalker, V.J., Owen, W.M., Paterson, C., Barker, E., Brooks, H., Rycroft, A.N., Brownlie, J., 2004. Mycoplasmas associated with canine infectious respiratory disease. Microbiology 150, 3491-3497.

Chan, C.M., Ridgway, M.D., Mitchell, M.A., Maddox, C.W., 2013. Association between Mycoplasma-specific polymerase chain reaction assay results and oral bacterial contamination of bronchoalveolar lavage fluid samples from dogs with respiratory tract disease: 121 cases (2005-2012). Journal of the American Veterinary Medical Association 243, 1573-1579.

Chandler, J.C., Lappin, M.R., 2002. Mycoplasmal respiratory infections in smal animals: 17 cases (1988-1999). Journal of the American Animal Hospital Association 38, 111-119.

Clercx, C., Peeters, D., Snaps, F., Hansen, P., McEntee, K., Detilleux, J., Henroteaux, M., Day, M.J., 2000. Eosinophilic bronchopneumopathy in dogs. Journal of Veterinary Internal Medicine 14, 282-291.

Clercx, C., Peeters, D., German, A.J., Khelil, Y., McEntee, K., Vanderplasschen, A., Schynts, F., Hansen, P., Detilleux, J., Day, M.J., 2002. An immunologic investigation of canine eosinophilic bronchopneumopathy. Journal of Veterinary Internal Medicine 16, 229-237.
Clercx, C., Peeters, D., 2007. Canine eosinophilic bronchopneumopathy. Veterinary Clinics of North America: Small Animal Practice 37, 917-935.

Corcoran, B.M., Thoday, K.L., Henfrey, J.I., Simpson, J.W., Burnie, A.G., Mooney, C.T., 1991. Pulmonary infiltration with eosinophils in 14 dogs. Journal of Small Animal Practice 32, 494-502.

Ennis, D.P., Cassidy, J.P., Mahon, B.P., 2004. Prior Bordetella pertussis infection modulates allergen priming and the severity of airway pathology in a murine model of allergic asthma. Clinical \& Experimental Allergy 34, 1488-1497.

El Sayed Zaki, M., Raafat, D., El Metaal, A.A., 2009. Relevance of serology for Mycoplasma pneumoniae diagnosis compared with PCR and culture in acute exacerbation of bronchial asthma. American Journal of Clinical Pathology 131, 74-80.

Hansbro, P.M., Horvat, J.C., Gibson, P.G., 2004. Role of atypical bacterial infection of the lung in predisposition/protection of asthma. Pharmacology \& Therapeutics $101,193-210$.

Harju, T.H., Leinonen, M., Nokso-Koivisto, J., Korhonen, T., Räty, R., He, Q., Hovi, T., Mertsola, J., Bloigu, A., Rytilä, P., et al., 2006. Pathogenic bacteria and viruses in induced sputum or pharyngeal secretions of adults with stable asthma. Thorax 61, 579-584.

Iramain, R., Jara, A., Jimenez, J., Bogado, N., Cardozo, L., 2016. Chlamydia pneumoniae, and Mycoplasma pneumoniae: are they related to severe asthma in childhood? Journal of Asthma 53, 618-621.

Kavanagh, H., Noone, C., Cahill, E., English, K., Locht, C., Mahon, B.P., 2010. Attenuated Bordetella pertussis vaccine strain BPZE1 modulates allergen-induced immunity and prevents allergic pulmonary pathology in a murine model. Clinical \& Experimental Allergy 40, 933-941.

Kraft, M., Cassell, G.H., Pak, J., Martin, R.J., 2002. Mycoplasma pneumoniae and Chlamydia pneumoniae in asthma: effect of clarithromycin. Chest 121,1782-1788.

Lavan, R., Knesl, O., 2015. Prevalence of canine infectious respiratory pathogens in asymptomatic dogs presented at US animal shelters. Journal of Small Animal Practice 56, 572-576.

Mannering, S.A., McAuliffe, L., Lawes, J.R., Erles, K., Brownlie, J., 2009. Strain typing of Mycoplasma cynos isolates from dogs with respiratory disease. Veterinary Microbiology 135, 292-296.

Martin, R.J., Kraft, M., Chu, H.W., Berns, E.A., Cassell, G.H., 2001. A link between chronic asthma and chronic infection. Journal of Allergy and Clinical Immunology 107, 595-601.

Medina, J.L., Coalson, J.J., Brooks, E.G., Winter, V.T., Chaparro, A., Principe, M.F., Kannan, T.R., Baseman, J.B., Dube, P.H., 2012. Mycoplasma pneumoniae CARDS toxin induces pulmonary eosinophilic and lymphocytic inflammation. American Journal of Respiratory Cell and Molecular Biology 46, 815-822.

Nicolai, A., Nenna, R., Stefanelli, P., Carannante, A., Schiavariello, C., Pierangeli, A., Scagnolari, C. Moretti, C., Papoff, P., Bonci, E., et al., 2013. Bordetella pertussis in infants hospitalized for acute respiratory sy $\mu$ ptoms remains a concern. BMC Infectious Diseases 13, 526-532.

Peeters, D., McKiernan, B.C., Weisiger, R.M., Schaeffer, D.J., Clercx, C., 2000. Quantitative bacterial cultures and cytological examination of bronchoalveolar lavage specimens in dogs. Journal of Veterinary Internal Medicine 14, 534-541.

Peeters, D., Clercx, C., Day, M.J., 2005. Distribution of leucocyte subsets in bronchial mucosa from dogs with eosinophilic bronchopneumopathy. Journal of Comparative Pathology 133, 128-135.

Priestnall, S.L., Mitchell, J.A., Walker, C.A., Erles, K., Brownlie, J., 2014. New and emerging pathogens in canine infectious respiratory disease. Veterinary Pathology 51, 492-504.

Rajamäki, M.M., Järvinen, A.K., Sorsa, T., Maisi, P., 2002. Clinical findings, bronchoalveolar lavage fluid cytology and matrix metalloproteinase-2 and -9 in canine pulmonary eosinophilia. Veterinary Journal 163, 168-181.

Rozanski, E., 2014. Canine chronic bronchitis. Veterinary Clinics of North America: Small Animal Practice 44, 107-116.

Ruch-Gallie, R., Moroff, S., Lappin, M.R., 2016. Adenovirus 2, Bordetella bronchiseptica, and parainfluenza molecular diagnostic assay results in puppies after vaccination with modified live vaccines. Journal of Veterinary Internal Medicine 30, 164-166.

Rycroft, A.N., Tsounakou, E., Chalker, V., 2007. Serological evidence of Mycoplasma cynos infection in canine infectious respiratory disease. Veterinary Microbiology 120, 358-362.

Schulz, B., Kurz, S., Weber, K., Balzer, H., Hartmann, K., 2014. Detection of respiratory viruses and Bordetella bronchiseptica in dogs with acute respiratory tract infections. Veterinary Journal 201, 365-369.

Wakashin, H., Maezawa, Y., Kagami, S., Suto, A., Watanabe, N., Saito, Y., Hatano, M. Tokuhisa, T., Iwakura, Y., Puccetti, P., et al., 2008. IL23 and Th17 celles enhance TH2-cell mediated eosinophilic airway inflammation in mice. American Journal of Respiratory and Critical Care Medicine 178, 1023-1032.

Ye, Q., Xu, X.J., Shao, W.X., Pan, Y.X., Chen, X.J., 2014. Mycoplasma pneumonia infection in children is a risk factor for developing allergic diseases. The Scientific World Journal 2014, 1-11 ID 9865277.

Yin, S.S., Ma, F.L., Gao, X., 2017. Association of Mycoplasma pneumoniae infection with increased risk of asthma in children. Experimental and Therapeutic Medicine 13, 1813-1819.

Zeugswetter, F., Weissenböck, H., Shibly, S., Hassan, J., Spergser, J., 2007. Lethal bronchopneumonia caused by Mycoplasma cynos in a litter of golden retriever puppies. Veterinary Record 161, 626-627. 